\title{
Monitoring long distance WDM communication lines using a high-loss loopback supervisory system
}

\author{
Mousaab M. Nahas and Keith J. Blow
}

\section{Abstract}

In this paper, we present experimental results for monitoring long distance WDM communication links using a line monitoring system suitable for legacy optically amplified long-haul undersea systems. This monitoring system is based on setting up a simple, passive, low cost high-loss optical loopback circuit at each repeater that provides a connection between the existing anti-directional undersea fibres, and can be used to define fault location. Fault location is achieved by transmitting a short pulse supervisory signal along with the WDM data signals where a portion of the overall signal is attenuated and returned to the transmit terminal by the loopback circuit. A special receiver is used at the terminal to extract the weakly returned supervisory signal where each supervisory signal is received at different times corresponding to different optical repeaters. Therefore, the degradation in any repeater appears on its corresponding supervisory signal level. We use a recirculating loop to simulate a $4600 \mathrm{~km}$ fibre link, on which a high-loss loopback supervisory system is implemented. Successful monitoring is accomplished through the production of an appropriate supervisory signal at the terminal that is detected and identified in a satisfactory time period after passing through up to $45 \mathrm{~dB}$ attenuation in the loopback circuit.

\section{Acknowledgments}

The authors are thankful to all those who contributed to this work, namely: Stuart Barnes, Steve Webb, Stephen Desbrulais, John Ellison, Joerg Schwartz, Robin Ibbotson and Paul Harper.

\section{Introduction}

Line monitoring is required to diagnose the location of faults along any communication link [1]. The demand for optical line monitoring has greatly increased due to the high capacity and long distances of current undersea transmission links. These transmission links require continuous monitoring that should be carried out at the network terminal. Such remote monitoring enables instantaneous determination of any degradation along the fibre link which in turn allows rapid maintenance execution to be performed. In fact, the main problem with monitoring legacy optically amplified links is that the system gives limited information about what is happening along the fibre link, which has not been the case in electrical systems or even regenerative optical systems. Those systems have much more complex electrical regenerators that carry out a huge amount of processing, thus they can give very detailed monitoring information (e.g. error-rates on individual channels). In other words, regenerative systems typically use information derived at the regenerators to monitor link performance where this information is then multiplexed onto the data stream and can be used at the network terminal to determine the fault location. On the other hand, the main requirement of the line monitoring system is that it should have a much lower cost than the transmission link itself; otherwise it is not a cost-effective solution. To meet such a requirement, several optical line monitoring techniques have been developed, where the most famous technique was based on coherent optical time domain reflectometry (COTDR) [2], [3], [4], [5], [6], [7] and [8]. The main advantage of COTDR is that it can define the fault at any part of the system rather than just the output from the repeaters as in other techniques. Nevertheless, this method still has a cost problem, since it performs an optical coherent detection, which is relatively expensive, rather than electrical coherent detection. It also has other drawbacks that it becomes difficult to measure for a very long fibre span, and the presence of optical isolators located in the optical amplifiers requires they get bypassed by couplers which transfer power between fibres [3]. Moreover, COTDR tends to be used out-of-service in the case of a fibre break because it needs a high optical power probe signal to yield sufficient Rayleigh backscattering for detection, which impairs the in-service data traffic channels. Other line monitoring methods have been demonstrated in [9], [10] and [11] but the main disadvantage of these methods is that they can only provide inservice monitoring. Therefore, in addition to the cost requirement, it is important to develop a line monitoring system that can be used both in-service and out-of-service, thus providing continuous monitoring of the transmission fibre link. These requirements have been met since 1996 by designing a relatively simple, low cost line monitoring technique that is particularly applicable to undersea optical transmission systems [12]. This method is based on the fact that the optical transmission system contains at least one optical fibre pair extending between two 
terminals. Each fibre has a sequence of fibre spans and optical repeaters (i.e. EDFAs). Each repeater can have a simple high-loss optical loopback circuit which provides a connection between the existing two fibres and can be used to define fault location. The loopback circuit taps off a portion of the optical signal propagating through one of the fibres, attenuates this portion and then returns it back to the terminal via another tap in the other fibre. This approach can be shown in Fig. 1.

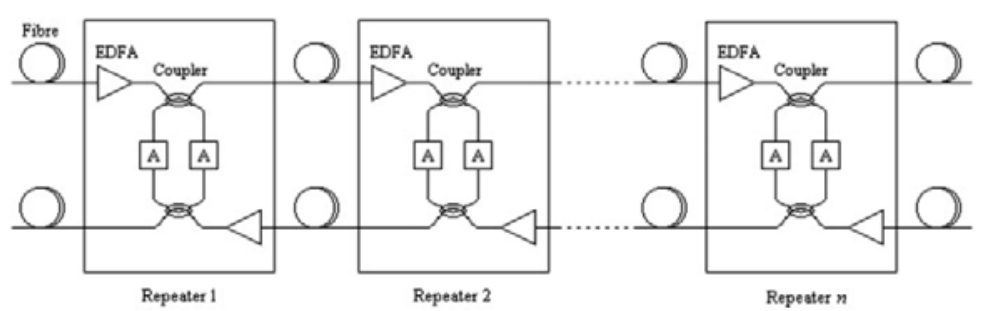

Fig. 1. Schematic diagram of the transmission fibre pair showing the high-loss loopback circuits.

Obviously, the loopback circuit can be very simple as it is composed of passive components which are two fibre couplers and two optical attenuators. All spans are assumed to be identical and the system is assumed to be symmetrical with respect to direction of propagation. Fault location can be determined by launching a supervisory signal into the fibre from one of the terminals, and returning the attenuated part of this signal to the terminal through the loopback circuit used at each repeater. Since the loopback circuit is passive, the repeaters do not return any information, all fault and location information must be gleaned from the returned signal levels and delays. It is essential to know that this method assumes that the main origin of the faults in the fibre link comes from impairments in the optical repeaters, specifically due to a failure of the pump laser diodes of EDFAs. Therefore, the fault being monitored is effectively the low EDFA gain due to faulty pump. This of course presumes that the other components including fibre spans are well-protected against all degradation factors, which should be the case in all legacy optical systems. Furthermore, faults in amplifiers in the forward path are monitored at the transmit terminal, while faults in amplifiers in the backward path are monitored at the other far end terminal, thus two monitoring equipments must operate simultaneously using different supervisory wavelengths to ensure full dual-path monitoring.

This paper implements the above approach experimentally using a short pulse supervisory transmitter and receiver at one optical line end (terminal) as proposed in [13] and [14]. This transmitter-receiver system is called line monitoring equipment and referred to as LME in this paper. The LME transmitter generates a low-power short pulse supervisory signal with a dedicated wavelength to be transmitted along with the original data signals, while the LME receiver detects the returned signals from the loopback circuits and examines the received supervisory signal. Again, both transmitter and receiver are located at the same terminal, and the lot can be used at either end of a fibre link. The paper aims to test the feasibility of the high-loss loopback supervisory system described here on long-haul WDM transmission system. This includes the generation, transmission and recovery of a weak LME supervisory pulse returned by the high-loss loopback circuit and swamped by the noise of the backward traffic signals. The typical loss of the loopback circuit is $45 \mathrm{~dB}$ according to [12] including all combined losses of the attenuator and the two tap couplers (see Fig. 1). Therefore, the experimental work of this paper is said to be successful if the system is able to generate an appropriate LME pulse and recover it at the receiver after $45 \mathrm{~dB}$ loopback attenuation over the maximum distance required.

\section{LME transmitter and receiver}

The block diagram of the LME transmitter is shown in Fig. 2. It comprises WDM traffic signals over a given set of wavelengths. A supervisory wavelength is coupled alongside the aggregate traffic signals. In practice, the supervisory wavelength is chosen to be between the adjacent traffic wavelengths of either the co-propagating traffic or counterpropagating traffic, and ideally in the midway between the traffic signals. The supervisory channel comprises a pulsed laser, at which the pulse duration is chosen to be as long as possible, but sufficiently short such that returned 
signals from each repeater do not overlap [13]. Since the duration of a pulse is greater than the Er3+ metastable lifetime $(\tau)$, the pulse must be modulated to prevent attenuation by a saturated EDFA and this can be achieved using a sub-carrier frequency of $2 \mathrm{MHz}$.

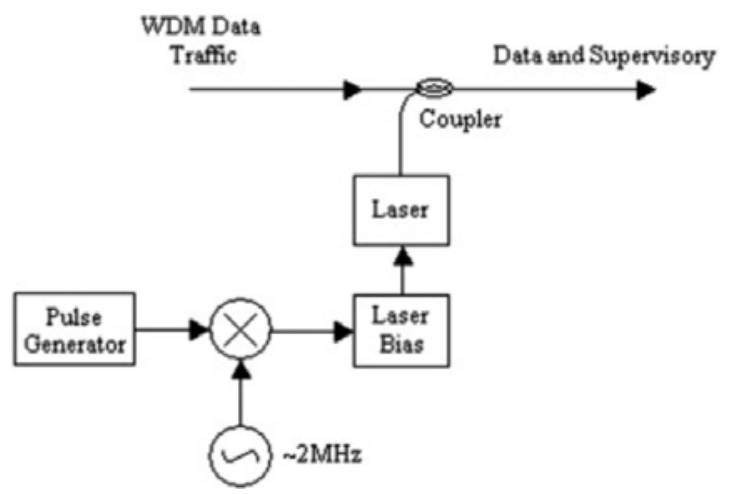

Fig. 2. Block diagram of the LME transmitter.

The block diagram of the LME receiver is shown in Fig. 3. It is a heterodyne receiver. It detects both the traffic and the supervisory signals looped back from each repeater. An optical band-pass filter is used to extract the supervisory signal and must be as narrow as possible (typically $\sim 0.2 \mathrm{~nm}$ in this case) to minimise crosstalk from adjacent traffic channels and to minimise ASE noise incident on the PIN. The detected signal from the PIN is passed through a lowpass filter of a few $\mathrm{kHz}$ bandwidth and then integrated. Integration is performed sequentially and the output from the ith integrator represents the supervisory signal level returned from the ith repeater, thus a set of parallel detectors is not required [14].

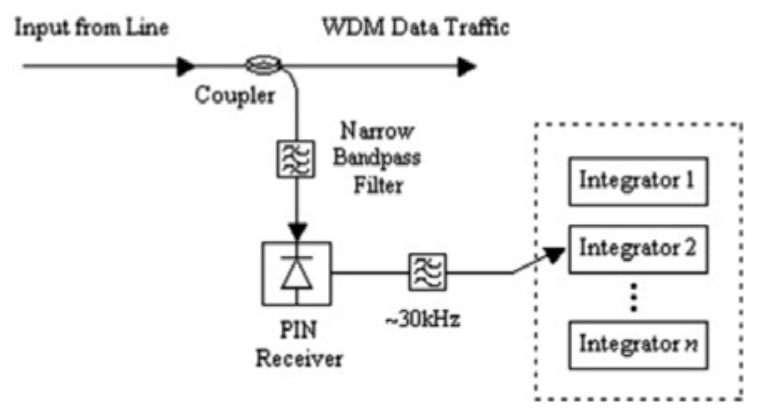

Fig. 3. Block diagram of the LME receiver.

This LME system allows an in-service measurement time to be in the order of 1 minute and is referred to as out-ofband LME system since the LME wavelength exists between the adjacent traffic wavelengths. In the original proposal [12], the LME signal is being superimposed over the data traffic signals, thus long integration times are required at the receiver to extract the small supervisory signal from the data signals, resulting in a measurement time of $\sim 4$ hours [13]. That approach is referred to as in-band LME system as the LME signal exists within a data signal band.

\section{Experimental setup}

This section discusses the experimental setup of the test bed used in the laboratory to simulate the system described in Fig. 1 on which the LME supervisory system is being examined.

\subsection{High-loss loopback system setup}

The high-loss loopback system was simulated in the laboratory as shown in Fig. 4, and was used to monitor one direction of the fibre pair. WDM data signals are coupled with an LME supervisory signal that is generated by the LME transmitter to give the composite forward or co-propagating traffic. The co-propagating path was simulated by using a recirculating loop, in which a single loopback circuit with variable attenuators is placed to monitor the performance at the output of the last EDFA. This of course provides one repeater monitoring where further 
repeaters can be monitored by increasing the number of recirculations, thus simulating different repeater loopback circuits. In practice, the recirculating loop has to be set up such that its round-trip time is larger than the LME supervisory pulse-width to ensure full propagation of the LME pulse in the loop. Also, the loop should have one LME pulse propagating in every recirculation. This was achieved by triggering the loop with the LME pulse, and setting the fill-time of the loop such that the next LME pulse appears in the next propagation cycle.

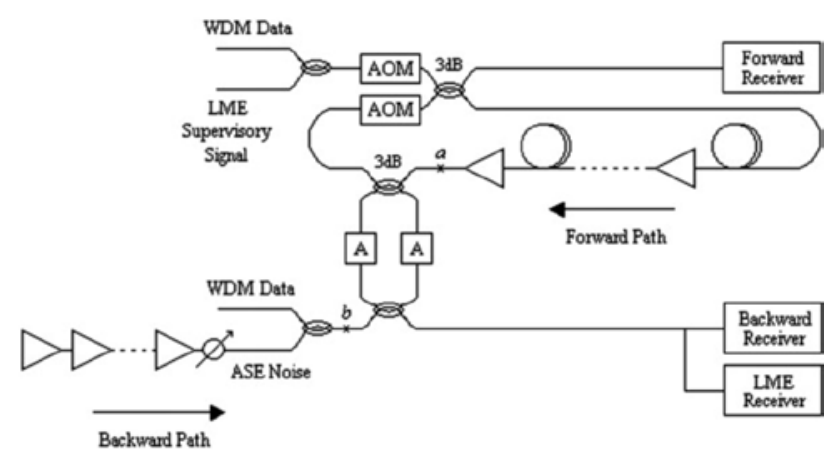

Fig. 4. High-loss loopback system setup.

In the counter-propagating direction, the backward path should have the counter-propagating data traffic signals along with ASE noise that includes both backward propagation noise and looped back noise. This indeed gives the ability to simulate the backward path without necessarily setting up fibre spans, but just by setting up a WDM data source and an ASE noise source, as appears in Fig. 4. The ASE noise source is composed of a chain of cascaded EDFAs followed by variable attenuator that is used to match the noise signal level to that in the co-propagating path. Therefore the noise at point $b$ in Fig. 4 must be equal to the noise at point $a$. The data signals are coupled with the noise signal by fibre coupler to give the composite backward traffic. Again, although the system setup does not simulate a full dual path system, which requires setting up two recirculating loops, it provides a test bed suitable for this experiment as long as the returned supervisory signal is swamped by the noise of a backward path which is in real system identical to that of the forward path, assuming that the system is symmetric.

\subsection{Complete experimental setup}

The entire setup for the WDM communication line monitoring experiment is shown in Fig. 5 . In the forward path, an LME transmitter produces optical LME supervisory pulses with duration of $500 \mu \mathrm{s}$, repetition rate of $8 \mathrm{~Hz}$, and carrier frequency of $2 \mathrm{MHz}$. The LME supervisory signal is then coupled with two WDM data signals in RZ modulation format and $10 \mathrm{Gbit} / \mathrm{s}$ bit-rate. These data signals were generated by using two LiNbO3 modulators driven by clock and data signals of a pattern generator with $10.709 \mathrm{GHz}$ frequency and 231-1 PRBS data. The wavelength of the data signals $\lambda 1$ and $\lambda 2$ are 1556.4 and $1557.4 \mathrm{~nm}$, respectively, while the LME signal wavelength is $1558.4 \mathrm{~nm}$, using DFB lasers in all channels. The reason for choosing such wavelengths is that the maximum backward noise from the noise source was achieved at around $1558.4 \mathrm{~nm}$, and the data signals are intended to be $1 \mathrm{~nm}$ apart from the LME signal and from each other. A variable optical attenuator follows the LME transmitter to adjust the LME signal level, which is supposed to be much lower than the data signal levels, typically $10 \mathrm{~dB}$ lower. The resultant data and LME signals are amplified to the required level and then propagate through a $200.9 \mathrm{~km}$ recirculating loop. The experiment starts with low power levels, which are $0 \mathrm{dBm}$ in each data signal and - $10 \mathrm{dBm}$ in LME signal at the input of the load AOM (or input $A O M)$. The launch powers into the fibre are hence lower by $6 \mathrm{~dB}$ due to the insertion losses of the $A O M$ and coupler. 


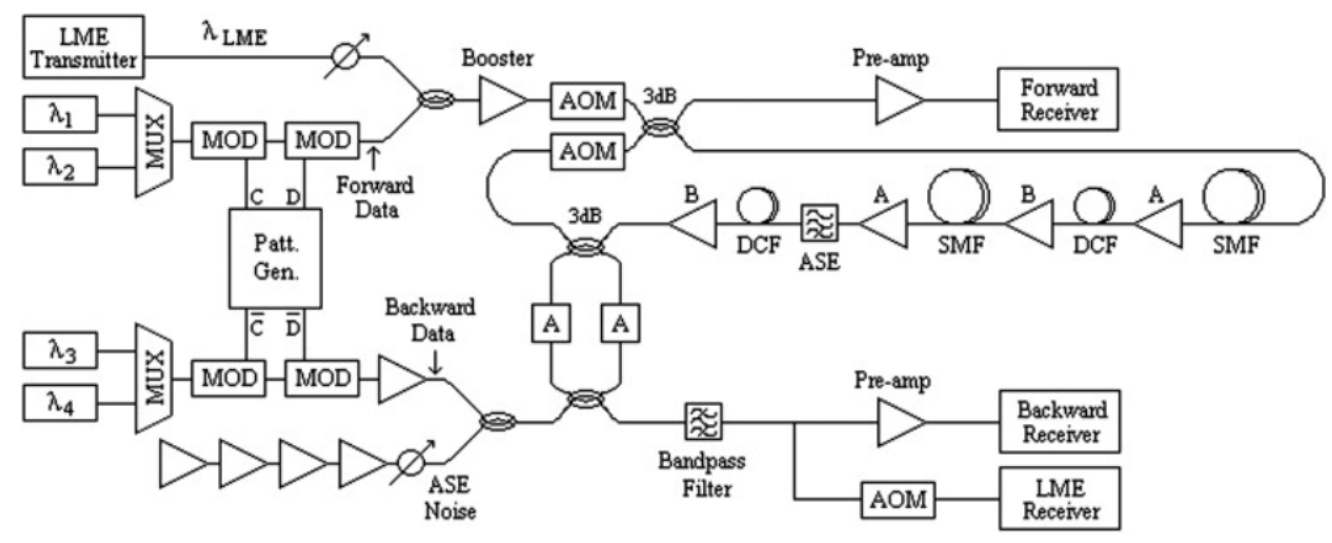

Fig. 5. A complete line monitoring experimental setup.

The recirculating loop itself consists of two SMF-DCF fibre sections. The first section consists of $82.5 \mathrm{~km}$ of SMF with $0.2 \mathrm{~dB} / \mathrm{km}$ attenuation coefficient and dispersion of $17 \mathrm{ps} / \mathrm{nm} / \mathrm{km}$ at $1550 \mathrm{~nm}$, and $15.2 \mathrm{~km}$ of DCF with attenuation of $0.5 \mathrm{~dB} / \mathrm{km}$ and a negative dispersion of $1387 \mathrm{ps} / \mathrm{nm}$. The second section consists of $86.7 \mathrm{~km}$ of SMF, and $16.5 \mathrm{~km}$ of DCF with attenuation of $0.5 \mathrm{~dB} / \mathrm{km}$ and a negative dispersion of $1383 \mathrm{ps} / \mathrm{nm}$. The losses of these fibres were compensated by using two C-band EDFAs in each section. The EDFAs denoted A in Fig. 5 had a $30 \mathrm{~dB}$ maximum small signal gain and $\sim 13 \mathrm{dBm}$ maximum output saturation power, while the EDFAs denoted $B$ had a $40 \mathrm{~dB}$ maximum small signal gain and $\sim 15 \mathrm{dBm}$ maximum output saturation power. The gain of each amplifier was set to compensate for the loss of the previous span, except for the last amplifier whose gain was increased to further compensate for the losses of the loopback tap coupler as well as the loop AOM and following coupler. All EDFAs had a noise figure of approximately $5 \mathrm{~dB}$. An ASE filter with an insertion loss of $3 \mathrm{~dB}$ was used before the last amplifier to remove the accumulated ASE gain peak at $1530 \mathrm{~nm}$. This $3 \mathrm{~dB}$ loss was compensated by the third amplifier, so power at the input of each fibre is constant.

In the backward path, two WDM data signals with wavelengths $\lambda 3$ and $\lambda 4$ of 1559.4 and $1560.4 \mathrm{~nm}$, respectively, are generated in the same way as those in the forward direction, using the same pattern generator but with the inverse clock and data signals. Four cascaded EDFAs; each with a noise figure of approximately $5 \mathrm{~dB}$ are set up to produce the backward noise. The gains of the EDFAs are set at the maximum while a variable attenuator is used to adjust the noise level corresponding to the transmission distance used in the forward direction. The noise signal is then coupled with the backward data signals to give the composite backward traffic that passes through the tap coupler of the loopback circuit. Recall that the power of the backward traffic must be similar to that of the forward traffic, i.e. each backward signal has a power of $-10 \mathrm{dBm}$ after the loopback coupler. Before the receiver, a tuneable band-pass filter with $0.24 \mathrm{~nm}$ bandwidth and $7 \mathrm{~dB}$ insertion loss is used to extract the supervisory wavelength to be detected. The filter can then be used as a demultiplexer for the backward data signals that can be therefore pre-amplified and detected by the sampling oscilloscope, or to extract the LME signal that can be detected and examined by the LME receiver. Note that for examining the forward traffic, the band-pass filter and the pre-amplifier are also required at the output of the recirculating loop as shown on the top of Fig. 5. However, before detecting the LME signal, it has to pass through an AOM being triggered with the recirculating loop to ensure that the LME receiver will detect the LME pulse of the last recirculation, while the other recirculated pulses are eliminated. This implies that the different LME pulses that correspond to different repeaters are detected and examined individually, unlike the real system in which they are detected and displayed serially according to their receiving times. This is a result of the fact that there is only one loopback circuit in the experiment which is used to monitor one repeater at a time. The LME receiver has a local frequency of $2.455 \mathrm{MHz}$, receiver sensitivity of $-60 \mathrm{dBm}$ and electrical bandwidth of $6.3 \mathrm{kHz}$. 


\section{Results and analysis}

\subsection{Recirculating loop measurements}

Before running the line monitoring test, it was essential to measure the OSNR versus distance for the forward data signals to determine the distance limit of the system. Fig. 6 presents the OSNR and noise measurements as a function of transmission distance for the forward data signal $(\lambda 1=1556.4 \mathrm{~nm})$ using an OSA resolution bandwidth of $0.5 \mathrm{~nm}$. It can be seen that data signals can propagate over $\sim 4600 \mathrm{~km}$ with acceptable OSNR performance, and this distance can be considered the limit of our transmission link. This would imply that the high-loss loopback supervisory system is to be examined over $4600 \mathrm{~km}$. (Note: similar performance was obtained for the other forward data signal $(\lambda 2=1557.4 \mathrm{~nm})$ ).

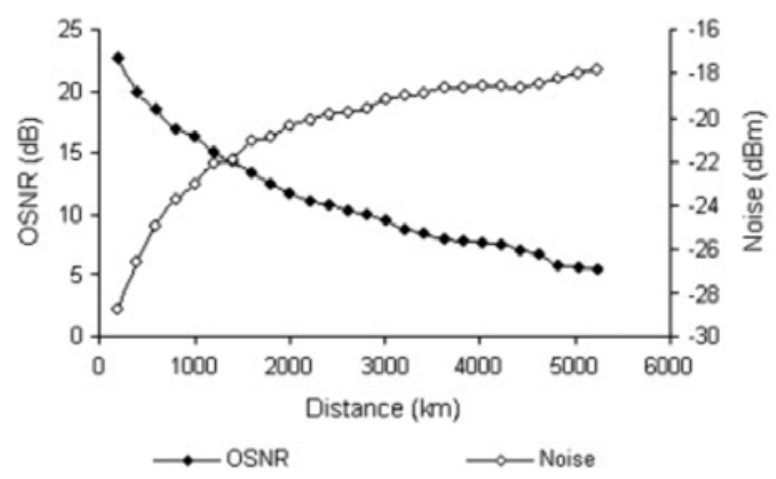

Fig. 6. OSNR and noise versus transmission distance for data signal $(\lambda 1=1556.4 \mathrm{~nm})$.

Furthermore, the noise measurements over distance in Fig. 6 were also used to measure how much ASE noise should be generated in the backward direction by the four cascaded EDFAs (see Fig. 5). Obviously, the level of the backward noise was varied for different numbers of recirculations according to the noise level in the forward direction. It is important to remember that these noise measurements were taken at the output of the recirculating loop, which means that they had already been attenuated by the losses of the loop AOM and the $3 \mathrm{~dB}$ coupler. Referring to Fig. 4 , since the noise at point $b$ must equal the noise at point $a$, the noise values of Fig. 6 must be offset by $+9 \mathrm{~dB}$. This amount includes the $3 \mathrm{~dB}$ loss of the loopback coupler in the forward direction in addition to the $6 \mathrm{~dB}$ of the AOM and its following coupler. Given this, the backward noise can be simply achieved by setting the four EDFAs at their maximum gain, and varying the optical attenuator to produce the required amount of noise. Note that the noise level refers to the level of the ASE noise that was observed near the LME wavelength.

The power evolution of the data and LME signals as a function of transmission distance is shown in Fig. 7. Since the power was measured at the output of the loop, it was attenuated by $6 \mathrm{~dB}$ through the loop AOM and the coupler. However, it was seen that the power level of both signals drops down as the distance increases, where it reduces by almost $3 \mathrm{~dB}$ after $4600 \mathrm{~km}$. This is due to the build up of ASE noise while the total average power remains constant. It can also be seen that the LME signal drops below the noise level after $2600 \mathrm{~km}$ and could not be measured.

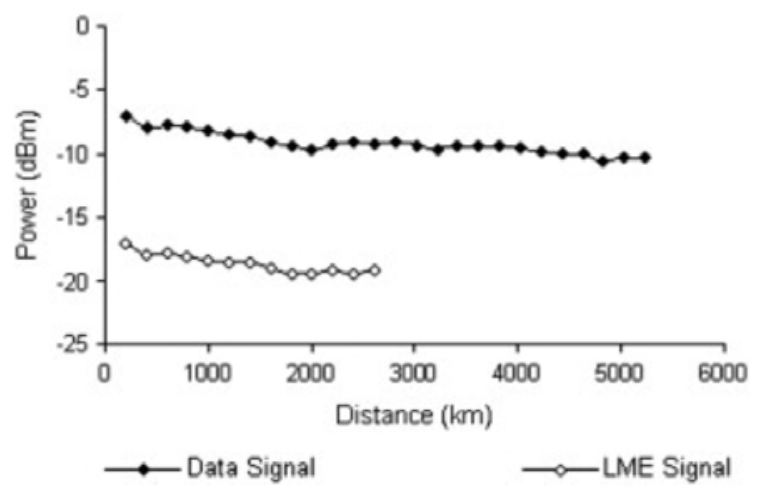

Fig. 7. Power versus transmission distance for data signal $(\lambda 1=1556.4 \mathrm{~nm})$ and the LME signal. 


\subsection{Line monitoring results}

Having set up both forward and backward paths, a monitoring test can be performed. As mentioned, the experiment aimed to use the high-loss loopback supervisory system described above to produce, transmit and detect/recover the attenuated LME supervisory pulse that coexists with the high noise of the backward traffic. Recall that the LME pulse has been transmitted as an envelope of a $2 \mathrm{MHz}$ sub-carrier frequency. In practice, detection of the LME carrier envelope is accomplished through an IQ demodulator used at the LME receiver. The amplitude of the

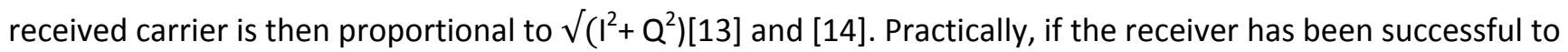
recover the LME pulse, then $\mathrm{I}, \mathrm{Q}$ and $\left(\mathrm{I}^{2}+\mathrm{Q}^{2}\right)$ curves are visible on the oscilloscope. Otherwise, the $\mathrm{LME}$ signal is lost or the receiver cannot extract it from the noise signals received with the LME signal. It is also important to remember that the LME pulse of different repeaters can be displayed with different times according to the repeater distance, e.g. the back-to-back pulse display is corresponding to the 0th repeater and so on.

As a result of running the experiment for different loopback attenuations, Fig. 8 shows a plot of the maximum transmission distance, at which the LME pulse is still being recovered, versus loopback attenuation.

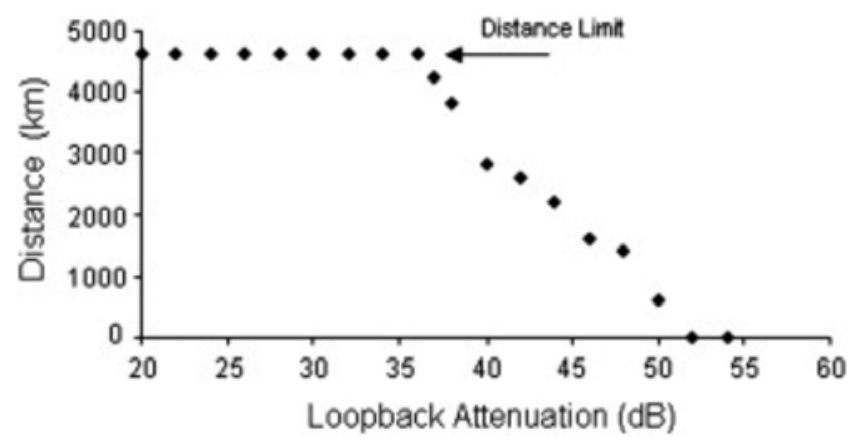

Fig. 8. Maximum transmission distance at which LME signal is being recovered versus loopback attenuation.

This graph means that, for instance, if the loopback attenuation is set at $40 \mathrm{~dB}$, the LME pulse can still be observed until the propagation distance reaches $2800 \mathrm{~km}$. Beyond this distance, the LME pulse is no longer visible on the oscilloscope. From the graph, it is also seen that the LME pulse is detectable over $4600 \mathrm{~km}$ if the loopback attenuation is less than or equal $36 \mathrm{~dB}$. Similarly, if the loopback attenuation is set to be $45 \mathrm{~dB}$ (the typical value), the LME signal cannot be detected over propagation distances larger than $1600 \mathrm{~km}$. Therefore, the challenge would be that the curve is shifted upward until the LME signal is successfully recovered over $4600 \mathrm{~km}$ using $45 \mathrm{~dB}$ loopback attenuation. To achieve this, an adjustment on the LME power level is required. While the LME signal had $-10 \mathrm{dBm}$ entering the load AOM, the power after the loopback coupler of the forward direction is also $-10 \mathrm{dBm}$ when the loop is balanced. This basically gives that the power is $-7 \mathrm{dBm}$ before the loopback coupler, i.e. at point a in Fig. 4 , which will enter the loopback circuit. From the signal evolution measurements presented in Fig. 7, the LME power drops by approximately $3 \mathrm{~dB}$ after $4600 \mathrm{~km}$. Having attenuated this signal by $36 \mathrm{~dB}$, which is the maximum loopback attenuation achieved for this system, the power of the looped back LME pulse becomes $-46 \mathrm{dBm}$. This weak signal is swamped by the backward noise signal with $-12.4 \mathrm{dBm}$ power according to the noise measured at $4600 \mathrm{~km}$ in Fig. 6, which was offset by $+9 \mathrm{~dB}$ but then attenuated by the loopback coupler of the backward path. Therefore, the LME signal that is received is lower than the backward noise signal by $\sim 34 \mathrm{~dB}$, although the composite signal is further attenuated by the band-pass filter and the final AOM. In addition, the LME signal is also accompanied by a portion of the forward noise signal which is also higher than the LME signal by about $2 \mathrm{~dB}$ after $4600 \mathrm{~km}$. If the loopback attenuation is increased by $1 \mathrm{~dB}$, the LME pulse cannot be seen on the oscilloscope at $4600 \mathrm{~km}$ as in Fig. 8. It is then possible to be seen at a distance that is shorter by almost two recirculations (i.e. $400 \mathrm{~km}$ ). In fact, this argument led to that the curve of Fig. 8 can be improved by simply increasing the LME signal power entering the system until the $45 \mathrm{~dB}$ loopback loss is achieved with $4600 \mathrm{~km}$. This would hence allow defining the minimum acceptable LME power for this system which can be used to obtain the aimed $45 \mathrm{~dB}$ loopback attenuation. Theoretically, the increase of the loopback attenuation is linearly proportional to the increase in the LME power. Practically, this was examined by running the same experiment for increased LME power levels and plotting curves similar to that of Fig. 8. As a result, 
we plotted the maximum possible loopback attenuation versus the LME signal power over $4600 \mathrm{~km}$ as shown in Fig. 9. The curve shows linear improvement of loopback attenuation with LME signal power with a slope of 1.15, i.e. $1 \mathrm{~dB}$ increase in the LME power results in $1.15 \mathrm{~dB}$ improvement in the loopback attenuation.

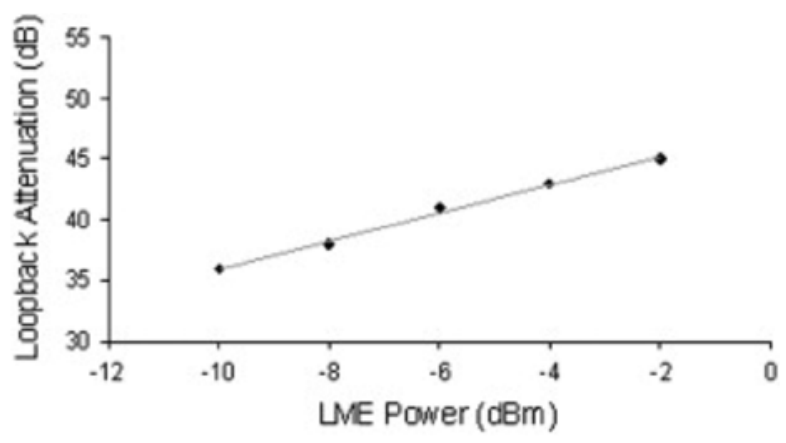

Fig. 9. The loopback attenuation versus LME power over $4600 \mathrm{~km}$ propagation distance.

From Fig. 9, it can be seen that the loopback attenuation of $45 \mathrm{~dB}$ is achieved experimentally when the LME signal power is $-2 \mathrm{dBm}$ at the input to the load AOM. The LME signal power launched into the fibre span of the loop is then $-8 \mathrm{dBm}$ due to $3 \mathrm{~dB}$ loss in the AOM and coupler, while the data signal powers are $0 \mathrm{dBm}$. At this level the LME signal power had no effect on the WDM data signals' performance. Ideally, the ratio between the data signal power and the LME signal power should not be less than $6 \mathrm{~dB}$, which has been met in this experiment.

Moreover, it is also essential to evaluate the performance of the LME receiver through the measurement of the electrical signal-to-noise ratio (eSNR) of the detected LME signal. Recall that the LME signal is swamped by the noise and entirely disappears in the optical domain after long distance, resulting in negative OSNR if measurable. As mentioned above, the received OSNR at the input to the LME receiver would be in the region of $-34 \mathrm{~dB}$. In this regime, the eSNR is mostly dictated by the optical noise received rather than electrical receiver noise. The eSNR can then be theoretically obtained by using:

eSNR $=\frac{P_{\text {Sig }}}{P_{\text {Nose }}}=\frac{\left|A_{\text {Sig }}\right|^{2}}{\sigma}$

where A Sig is the amplitude of the LME pulse and $\sigma$ is the standard deviation. Since the received LME pulse has

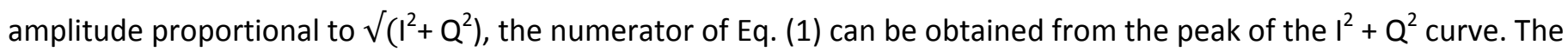
denominator can be calculated from the variance between I and $Q$. Therefore, the measurement of the eSNR can be approximately expressed as:

$\operatorname{eSNR}=\frac{\operatorname{Ave}\left(\mathrm{I}^{2}+\mathrm{Q}^{2}\right)}{\operatorname{Var}(\mathrm{I}, \mathrm{Q})}$

where the $\mathrm{I}^{2}+Q^{2}$ peak is averaged over a small region around the maximum value, and the $I$ and $Q$ variance is measured over a wider region in the zero level.

Practically, the eSNR was measured from the $\mathrm{I}, \mathrm{Q}$ and $\mathrm{I}^{2}+\mathrm{Q}^{2}$ curves, and Fig. 10 shows the measurements (in $\mathrm{dB}$ ) versus distance for the returned LME signal using the optimal $-2 \mathrm{dBm}$ LME signal launch power and $45 \mathrm{~dB}$ loopback attenuation. In fact, this presentation of results for different distances simulates a serial detection of LME pulses corresponding to different repeaters in real system. It is seen that the eSNR of the LME signal reduces over distance and becomes $\sim 14 \mathrm{~dB}$ after $4600 \mathrm{~km}$. These measurements can be used to give indication about the overall performance of the LME receiver i.e. sensitivity and capability in detecting the week LME pulse after being attenuated and swamped by optical noise through transmission. 


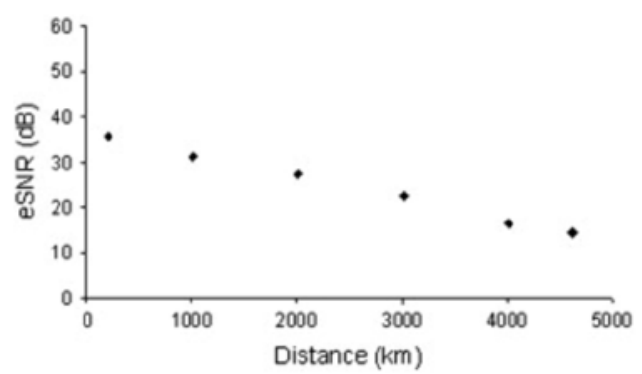

Fig. 10. eSNR measurements versus propagation distance for the LME signal using $-2 \mathrm{dBm}$ launch power and $45 \mathrm{~dB}$ loopback attenuation.

\section{Conclusions}

This paper has demonstrated the experimental characterisation for a monitoring system in a $4600 \mathrm{~km}$ WDM communication line using a high-loss loopback supervisory technique that is suitable for optically amplified long-haul undersea systems. This supervisory system is based on setting up a simple, passive, low cost high-loss optical loopback circuit at each repeater, where the loopback circuit provides a connection between the existing two undersea fibres and can be used to define fault location. Fault location can be obtained by transmitting a supervisory signal (referred to as an LME signal) along with the WDM data signals where a portion of the overall signal is attenuated and returned to the transmit terminal by the loopback circuit. A special receiver then extracts the weakly returned LME signal and displays it on the oscilloscope within a satisfactory measurement time ( 1 minute). The different LME pulses then correspond to different optical repeaters where the degradation in any repeater appears on its corresponding LME signal level. The entire high-loss loopback monitoring system presented in this paper has been successful in term of producing, transmitting and recovering a low-power pulsed supervisory signal that has been attenuated by the typical $45 \mathrm{~dB}$ loss in a loopback circuit and propagated over $4600 \mathrm{~km}$ using a recirculating loop. Achieving this result required adjusting the minimum LME signal power entering the WDM transmission link, while keeping the supervisory signal significantly lower than the WDM data signals. The performance of the LME receiver has also been evaluated through the measurement of the electrical signal-to-noise ratio (eSNR) of the detected LME signal for different numbers of recirculations hence simulating different repeaters monitoring results.

\section{References}

[1] I.P. Kaminow, T. Li, Optical Fibre Telecommunications IVB: Systems and Impairments, Academic Press, San Diego, London (2002)

[2] J.P. King, D.F. Smith, K. Richards, P. Timson, R.E. Epworth, S. Wright, Journal of Lightwave Technology, 5 (1987), p. 616

[3] Y. Horiuchi, S. Ryu, K. Mochizuki, H. Wakabayashi, IEEE Photonics Technology Letters, 2 (1990), p. 291

[4] Y. Sato, K. Aoyama, IEEE Photonics Technology Letters, 3 (1991), p. 1001.

[5] Y. Sato, K. Aoyama, Journal of Lightwave Technology, 10 (1992), p. 78, ,

[6] J.C. MacKichan, J.A. Kitchen, C.W. Pitt, Electronics Letters, 28 (1992), p. 626

[7] M. Sumida, S.I. Furukawa, K. Tanaka, M. Aiki, Journal of Lightwave Technology, 14 (1996), p. 2108

[8] P. Kim, H. Yoon, N. Park, J. Seo, K. Jeong, K. Ryoo, K. Lee, J. Blow, S. Fleming, Optical Fibre Technology, 8 (2002), p. 139

[9] J. Prat, S. Ruiz-Moreno, Optics Communications, 183 (2000), p. 65

[10] C.K. Chan, L.K. Chen, F. Tong, D. Lam, IEEE Photonics Technology Letters, 9 (1997), p. 1520

[11] C. Yeh, S. Chi, Optics Communications, 257 (2006), p. 306

[12] C. D. Anderson, "Supervisory apparatus for optical transmission system", U.S. Patent 5,825,515, (filed May 1996)

[13] S. Desbruslais, J. Ellison, D. Powell, “Short pulse loop back supervisory system”, UK Patent GB 2413447 A, (filed April 2005)

[14] S. Desbruslais, J. Ellison, D. Powell, "Pulsed high loss loop back signaling scheme”, UK Patent GB 2413447 B, (filed April 2005) 